EPJ Web of Conferences 107, 08007 (2016)

DOI: $10.1051 /$ epjconf/201610708007

(C) Owned by the authors, published by EDP Sciences, 2016

\title{
Analysis of proton scattering of stable and exotic light nuclei using an energy-dependent microscopic optical potential
}

\author{
H. M. Maridi ${ }^{1,2, a}$, M. Y. H. Farag ${ }^{1}$, and E. H. Esmael ${ }^{1}$ \\ ${ }^{1}$ Physics Department, Faculty of Science, Cairo University, Cairo, Egypt \\ ${ }^{2}$ Physics Department, Faculty of Applied Science, Taiz University, Taiz, Yemen
}

\begin{abstract}
The proton elastic scattering off the ${ }^{9,10,11,12} \mathrm{Be}$ isotopes at a wide energy range from 3 to 200 $\mathrm{MeV} /$ nucleon is analyzed using the optical model with the partial-wave expansion method. The microscopic optical potential (OP) is taken within the single-folding model. The density- and isospin-dependent M3YParis nucleon-nucleon $(N N)$ interaction is used for the real part and the $N N$-scattering amplitude of the highenergy approximation for the imaginary one. The cross-section data are reproduced well at energies up to 100 $\mathrm{MeV} /$ nucleon by use of the partial-wave expansion. For higher energies, the eikonal approximation is successfully used. The volume integrals of the OP parts have systematic energy dependencies and they can be parameterized as functions of energy. From these parametrization, an energy-dependent OP can be obtained.
\end{abstract}

\section{Introduction}

Study of light-nuclei properties is considered as an important and exciting research topic in modern nuclear physics. To understand the structure and reactions of the light nuclei, it is useful to study the cross sections of the proton elastic scattering on these nuclei.

In Ref. [1], we studied the cross sections for the proton elastic scattering of helium and lithium isotopes at incident energies below $160 \mathrm{MeV} /$ nucleon using the optical model analysis with the partial-wave expansion method. The results are in agreement with the data until to 100 $\mathrm{MeV} /$ nucleon.

In the present work, we present a microscopic analysis with the partial-wave expansion method for the cross-section data of proton elastic scattering off beryllium isotopes over a wide range of energies from few $\mathrm{MeVs}$ /nucleon up to $200 \mathrm{MeV} /$ nucleon. For $E \geq 50$ $\mathrm{MeV} /$ nucleon, the cross sections are recalculated by using the eikonal approximation that based on the Glauber theory and they are compared with the partial-wave expansion method. The theoretical approaches are given in Sec. 2, the results of the calculations are presented in Sec. 3, and a summary is given in Sec. 4 .

\section{Theoretical calculations}

The present problem considers a proton with an energy $E$ incident upon a target with a mass number $A$ and scattered by a microscopic spherical optical potential $U_{\mathrm{OP}}(r)$ which

\footnotetext{
ae-mail: h.maridi@gmail.com
}

can be written as [1-3]

$$
\begin{array}{r}
U_{O P}(r)=N_{R} V_{F}(r)+i\left[N_{I} W_{H}(r)-N_{\mathrm{IS}} r \frac{d}{d r} W_{H}(r)\right] \\
-2 \lambda_{\pi}^{2} N_{\mathrm{SO}} \frac{1}{r} \frac{d}{d r} V_{F}(r) \mathbf{L} \cdot \mathbf{S},
\end{array}
$$

where $V_{F}$ is the real OP using the single-folding model and $W_{H}$ is the volume imaginary OP using the high-energy approximation (HEA) model that was derived on the basis of the eikonal phase inherent in the optical limit of the Glauber theory. $N_{R}, N_{I}, N_{\text {IS }}$, and $N_{\text {SO }}$ are the renormalization factors that are introduced to fit the data. One can represent the direct and exchange parts of $V_{F}$ in terms of isoscalar $\left(V_{\mathrm{IS}}\right)$ and isovector $\left(V_{\mathrm{IV}}\right)$ contributions as [4]

$$
\begin{array}{r}
\begin{array}{r}
V_{F}(\mathbf{r})=V^{\mathrm{D}}(\mathbf{r})+V^{\mathrm{EX}}(\mathbf{r})=V_{\mathrm{IS}}^{\mathrm{D}}(\mathbf{r})+V_{\mathrm{IV}}^{\mathrm{D}}(\mathbf{r}) \\
+V_{\mathrm{IS}}^{\mathrm{EX}}(\mathbf{r})+V_{\mathrm{IV}}^{\mathrm{EX}}(\mathbf{r}),
\end{array} \\
V_{\mathrm{IS}(\mathrm{IV})}^{\mathrm{D}}(\mathbf{r})=\int\left[\rho_{p}\left(\mathbf{r}^{\prime}\right) \pm \rho_{n}\left(\mathbf{r}^{\prime}\right)\right] v_{00(01)}^{\mathrm{D}}(\rho, E, s) d^{3} r^{\prime}, \\
V_{\mathrm{IS}(\mathrm{IV})}^{\mathrm{EX}}(\mathbf{r})=\int\left[\rho_{p}\left(\mathbf{r}, \mathbf{r}^{\prime}\right) \pm \rho_{n}\left(\mathbf{r}, \mathbf{r}^{\prime}\right)\right] v_{00(01)}^{\mathrm{EX}}(\rho, E, s) \\
\times j_{0}(k(E, r) s) d^{3} r^{\prime},
\end{array}
$$

where the sign + is related to the isoscalar and the sign - to the isovector. Functions $v_{00(01)}^{\mathrm{D}}(\rho, E, s)$ and $v_{00(01)}^{\mathrm{EX}}(\rho, E, s)$ are the direct and exchange parts of the M3Y-Paris $N N$ interaction, where the subscripts 00 and 01 are denoted to the isoscalar and isovector components. The variable $s=$ $\left|\mathbf{r}^{\prime}-\mathbf{r}\right|$ is the distance between the proton and the nucleon in the target and $\mathbf{r}$ is the vector joining the center-of-mass of 
the incident proton and the target. The function $\rho_{p(n)}\left(\mathbf{r}, \mathbf{r}^{\prime}\right)$ is the one-body density matrix for the protons(neutrons) in the target nucleus with $\rho_{p(n)}(\mathbf{r}) \equiv \rho_{p(n)}(\mathbf{r}, \mathbf{r}) . k(E, r)$ is the local momentum of the relative motion. The forms of effective M3Y-Paris $N N$ interactions $v^{\mathrm{D}}$ and $v^{\mathrm{EX}}$, their energy and density dependencies, the realistic local approximation of the density matrix, as well as necessary explanations and values of calculation parameters can be found in Refs. [3, 4].

The imaginary OP using the HEA model is expressed in Ref. [5] as

$$
W_{H}(r)=-\frac{\hbar v}{(2 \pi)^{2}} \bar{\sigma}_{N N} \int_{0}^{\infty} d q q^{2} j_{0}(q r) \rho(q) f_{N N}(q)
$$

where $v$ is the velocity of the nucleon-nucleus relative motion, $\rho(q)$ is the form factor corresponding to the point-like nucleon density distribution of the nucleus, and $f_{N N}(q)$ is the $N N$ scattering amplitude. $\bar{\sigma}_{N N}$ is the average over isospin total $N N$ cross section. The Green's function Monte Carlo (GFMC) density [6] is used for ${ }^{9,10} \mathrm{Be}$ and the Gaussian-Oscillator (GO) distribution is used for ${ }^{11,12} \mathrm{Be}$ (see [3]).

The volume integrals are relatively invariant functions of the OP parameters and give insight in the behavior of the optical potentials as a function of mass and energy. The volume integrals per nucleon for the real, imaginary and spin-orbit parts of the OP are denoted by $J_{R}, J_{I}$, and $J_{\text {SO }}$, respectively. They are defined as

$$
\begin{gathered}
J_{R}=\frac{4 \pi}{A} \int\left[N_{R} V_{F}(r)\right] r^{2} d r \\
J_{I}=J_{\mathrm{IV}}+J_{\mathrm{IS}}=\frac{4 \pi}{A} \int\left[N_{\mathrm{IV}} W_{H}(r)-N_{\mathrm{IS}} r \frac{d}{d r} W_{H}(r)\right] r^{2} d r \\
J_{\mathrm{SO}}=\frac{4 \pi}{A} \int\left[-2 \lambda_{\pi}^{2} N_{\mathrm{SO}} \frac{1}{r} \frac{d}{d r} V_{F}(r)\right] r^{2} d r
\end{gathered}
$$

where $J_{\mathrm{IV}}$ and $J_{\mathrm{IS}}$ are the volume integrals of the volume and surface imaginary potentials, respectively.

The differential cross-section data of $p+{ }^{9,10,11,12} \mathrm{Be}$ elastic scattering and corresponding references are listed in Table 1 . In addition, the experimental values of the re-

Table 1. $d \sigma / d \Omega$ data of $p+{ }^{9,10,11,12}$ Be elastic scattering.

\begin{tabular}{ll}
\hline Scattering & Incident energy (in MeV/nucleon) [Reference] \\
\hline$p+{ }^{9} \mathrm{Be}$ & $3[7], 6.0,10.0[8], 13[9], 17,25[10], 30.3[9]$, \\
& $35.2[11], 49.4[12], 54.7,74.7[13], 100.6[14]$, \\
& $160[15], 179.9[16]$, and $201.4[17]$ \\
${ }^{10} \mathrm{Be}+p$ & $6,9[18], 12,15[19], 39.1[20]$, and $59.3[21]$ \\
${ }^{11} \mathrm{Be}+p$ & $38.4[20]$ and $49.3[21]$ \\
${ }^{12} \mathrm{Be}+p$ & $55[22]$ \\
\hline
\end{tabular}

action cross sections for $p+{ }^{9,10,11}$ Be reactions can be found in Refs. [23, 24]. The calculations of the cross sections are performed by use of the DWUCK4 code [25] for using the partial-wave expansion and by use of the DWEIKO code [26] for using the eikonal approximation. The renormalization factors of the OP are determined by a fitting procedure of the scattering observables which is carried out to achieve minimum $\chi^{2}$

$$
\chi^{2}=\frac{1}{N} \sum_{k=1}^{N}\left[\frac{\sigma_{\mathrm{th}}\left(\theta_{k}\right)-\sigma_{\mathrm{ex}}\left(\theta_{k}\right)}{\Delta \sigma_{\mathrm{ex}}\left(\theta_{k}\right)}\right]^{2},
$$

where $\sigma_{\text {th }}\left(\theta_{k}\right)$ and $\sigma_{\text {ex }}\left(\theta_{k}\right)$ are the theoretical and experimental cross sections at the angle $\theta_{k}$, respectively. $\Delta \sigma_{\text {ex }}\left(\theta_{k}\right)$ is the experimental error and $N$ is the number of data points. The errors of the experimental data are not provided for most of the considered data, so these errors can be taken as $10 \%$ of the corresponding data.

\section{Results and discussion}

The differential cross sections of $p+{ }^{9,10,11,12}$ Be elastic scattering are calculated using the partial-wave expansion and presented in Fig. 1. The best-fit renormalization factors are listed in Table II in our work [3]. The differential crosssection data for energies up to $100 \mathrm{MeV} /$ nucleon are reproduced well. For higher energies, more than one minimum are found in the calculated differential cross sections as shown in Fig. 1(b) for $p+{ }^{9}$ Be elastic scattering. At these high energies, the wave function will oscillate rapidly and the calculations of scattering wave functions for each partial wave become more complicated. Instead of the partialwave expansion, it is useful to use the eikonal approximation which is based on the optical limit of the Glauber theory [27]. Then, these cross sections at energies $\geq 50$ $\mathrm{MeV} /$ nucleon are recalculated using the eikonal approximation as presented in Fig. 1(b). The renormalization factors for the eikonal approximation calculations are listed in Table IV in our work [3]. It is clear that the eikonal approximation gives a good agreement with the data than the partial-wave expansion at energies $\geq 50 \mathrm{MeV} /$ nucleon over all the angular range.

The calculated reaction cross sections $\sigma_{R}$ for $p+{ }^{9,10,11,12} \mathrm{Be}$ at different energies using the optical model analysis with the partial-wave expansion are presented in Fig. 2(a). The $\sigma_{R}$ values obtained for the considered reactions are in agreement with the available experimental data. Furthermore, the calculated $\sigma_{R}$ values for the halo-nucleus reaction $\left({ }^{11} \mathrm{Be}+p\right)$ are found to be larger than the calculated ones for the reactions of their isotopes. In Fig. 2(b), the calculated values of $\sigma_{R}$ using the eikonal approximation for $p+{ }^{9} \mathrm{Be}$ reaction give good agreement with the experimental data than those results of the partial-wave expansion for energies $\geq 50 \mathrm{MeV} /$ nucleon.

The calculated volume integrals of the best-fit OPs for $p+{ }^{9,10,11,12} \mathrm{Be}$ elastic scattering are shown in Fig. 3. Clearly, the $J_{R}$ and $J_{\text {SO }}$ increase with energy until they reach a maximum at a definite energy (denoted by $E_{R}$ for $J_{R}$ and $E_{\mathrm{SO}}$ for $J_{\mathrm{SO}}$ ). After that, they begin to decrease with energy. The $J_{I}$ begins small at low energies and then increases rapidly up to a maximum value $\left(J_{\mathrm{I} 0}\right)$. Thereafter, it decreases linearly and slowly with increasing energy. Clearly, the greatest value for $J_{I}$ is found for the halo nucleus scattering $\left({ }^{11} \mathrm{Be}+p\right)$. There are different energy parameterizations for the calculated volume integrals. In the present work, the reciprocal formula [1] is expressed 

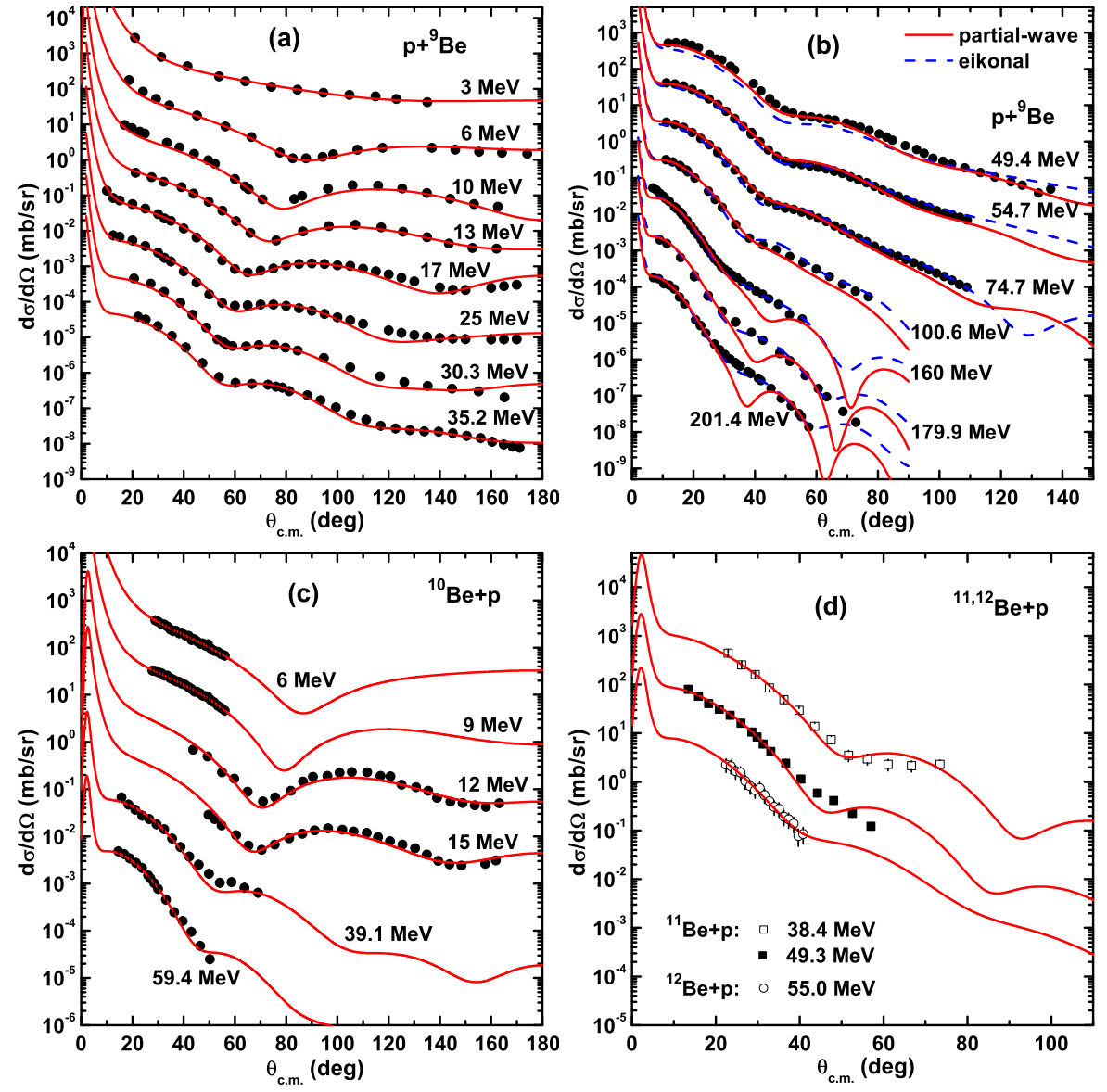

Figure 1. (Color online) Differential cross sections for $p+{ }^{9,10,11,12}$ Be elastic scattering. The symbols represent the experimental data and the lines represent the results of the calculations. The curves and data points at the top represent true values, while the others are offset by factors of 10,100 , and so on.
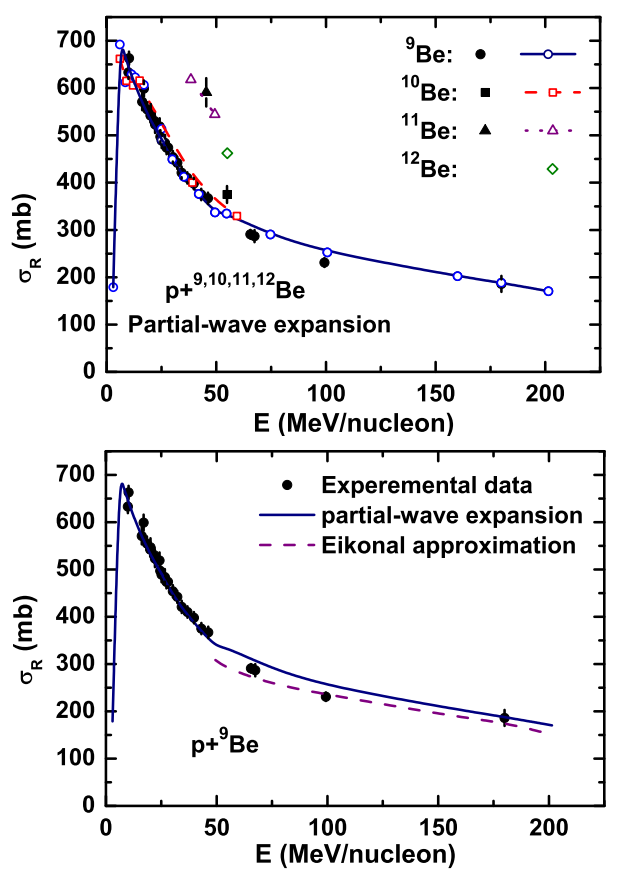

Figure 2. (Color online) Energy dependence of calculated $\sigma_{R}$ for $p+{ }^{9,10,11,12}$ Be elastic scattering. for both $J_{R}$ and $J_{\mathrm{SO}}$. For energies lower than $E_{R}$ and $E_{\mathrm{SO}}$, the Gaussian parametrization [28] is used.

$$
J_{R}(E)= \begin{cases}J_{\mathrm{R} 0} /\left(1+\eta_{R} E\right) & \text { for } E \geq E_{R} \\ J_{\mathrm{R} 1} \exp \left[-\left(E-E_{R}\right)^{2} / w_{R}^{2}\right] & \text { for } E \leq E_{R} .\end{cases}
$$

Similarly,

$$
J_{\mathrm{SO}}(E)= \begin{cases}J_{\mathrm{S} 0} /\left(1+\eta_{\mathrm{SO}} E\right) & \text { for } E \geq E_{\mathrm{SO}} \\ J_{\mathrm{S} 1} \exp \left[-\left(E-E_{\mathrm{SO}}\right)^{2} / w_{\mathrm{SO}}^{2}\right] & \text { for } E \leq E_{\mathrm{SO}},\end{cases}
$$

where $J_{k 1}=J_{k 0} /\left(1+\eta_{k} E_{k}\right)$ with $k=R$, SO. $J_{0}$ is the maximum value; $w$ is the rise parameter which describes the increasing of the $J_{R}$ and $J_{\mathrm{SO}}$ at low energies; $\eta$ is the decay parameter that describes a decrease after they reach their maximum values. For $J_{I}$, the modified Fermi parametrization formula [3] is used. It can be expressed as

$$
J_{I}(E)=J_{\mathrm{I} 0}\left(1-\eta_{I} E\right) \frac{1}{1+\exp \left(E_{I}-E\right) / w_{I}}
$$

with a maximum value $J_{\mathrm{I} 0}$ and the following global parameters: a decreasing slope $\eta_{I}$, a rise parameter $w_{I}$, and $E_{I}$. For the eikonal approximation that can be used at energies $\geq 50 \mathrm{MeV} /$ nucleon, the first lines in Eqs. (10) and (11) are 

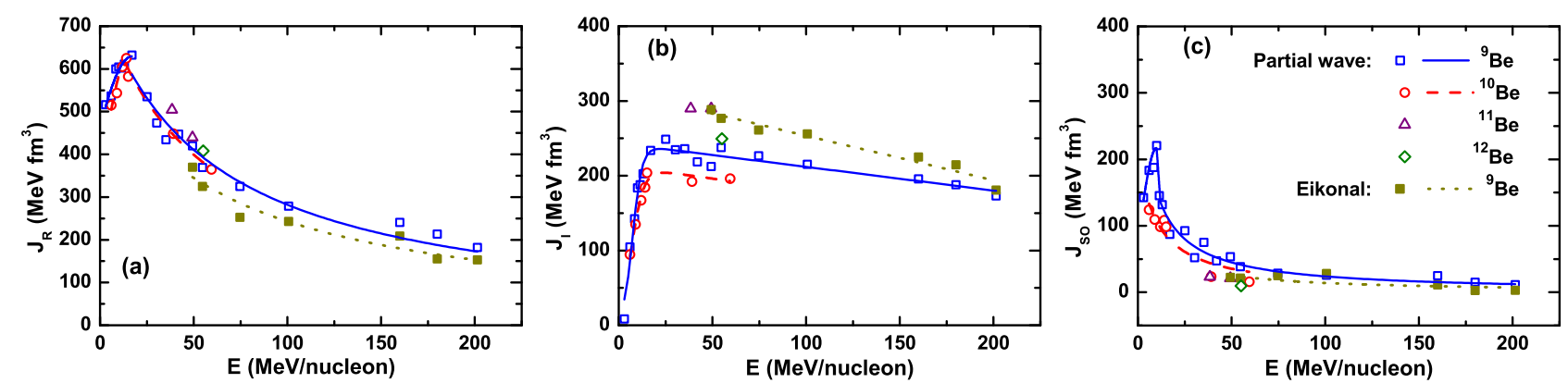

Figure 3. (Color online) Energy dependence of the volume integrals for $p+^{9,10,11,12} \mathrm{Be}$ elastic scattering. The symbols represent the calculated volume integrals and the lines represent the parameterized volume integrals as functions of energy.

considered only for $J_{R}$ and $J_{\mathrm{SO}}$. Also, the linear relation in Eq. (12) is only considered for $J_{I}$.

The parameterized volume integrals are shown in Fig. 3 in comparison with the calculated ones. The parameter values obtained for the considered reactions can be found in our work [3]. From these parameterizations, an energy-dependent OP can be obtained as explained in Ref. [2].

\section{Summary}

Microscopic analysis was performed for the proton elastic scattering off beryllium isotopes over a wide energy range from 3 to $201.4 \mathrm{MeV} /$ nucleon by using a folded OP. The cross sections were calculated using the optical model with the partial-wave expansion which successfully reproduces the data at energies until to $100 \mathrm{MeV} /$ nucleon. Above this energy value, some minima appear in the calculated differential cross sections. These cross sections are recalculated by use of the eikonal approximation which reproduces the cross-section data well over all the angular range. The calculated volume integrals have clear energy dependence and can be parameterized as functions of energy.

\section{References}

[1] M.Y.H. Farag, E.H. Esmael, and H.M. Maridi, Phys. Rev. C 88, 064602 (2013)

[2] M.Y.H. Farag, E.H. Esmael, and H.M. Maridi, Eur. J. Phys. A 50, 106 (2014)

[3] M.Y.H. Farag, E.H. Esmael, and H.M. Maridi, Phys. Rev. C 90, 034615 (2014)

[4] D.T. Khoa, E. Khan, G. Colò, and N. Van Giai, Nucl. Phys. A 706, 61 (2002)

[5] K.V. Lukyanov, V.K. Lukyanov, E.V. Zemlyanaya, A.N. Antonov, and M.K. Gaidarov, Eur. J. Phys. A 33, 389 (2007)

[6] Steven C. Pieper, K. Varga, and R.B. Wiringa, Phys. Rev. C 66, 044310 (2002)
[7] D.H. Loyd and W. Haeberli, Nucl. Phys. A 148, 236 (1970)

[8] F.W. Bingham, M.K. Brussel, and J.D. Steben, Nucl. Phys. 55, 265 (1964)

[9] H.J. Votava, T.B. Clegg, E.J. Ludwig, and W.J. Thompson, Nucl. Phys. A 204, 529 (1973)

[10] D.G. Montague, R.K. Cole, P.S. Lewis, C.N. Waddell, and D.L. Hendrie, Nucl. Phys. A 199, 433 (1973)

[11] E. Fabrici, S. Micheletti, M. Pignanelli, F.G. Resmini, R. De Leo, G. D'Erasmo, and A. Pantaleo, Phys. Rev. C 21, 844 (1980)

[12] N.M. Clarke, E.J. Burge, D.A. Smith, and J.C. Dore, Nucl. Phys. A 157, 145 (1970)

[13] L.J. de Bever et al., Nucl. Phys. A 579, 13 (1994)

[14] H. Seifert, Ph.D. thesis, University of Maryland, 1990.

[15] Philip G. Roos and N.S. Wall, Phys. Rev. 140, B1237 (1965)

[16] S. Dixit et al., Phys. Rev. C 43, 1758 (1991)

[17] H. Seifert et al., Phys. Rev. C 47, 1615 (1993)

[18] K.T. Schmitt et al., Phys. Rev. C 88, 064612 (2013)

[19] D.L. Auton, Nucl. Phys. A 157, 305 (1970)

[20] V. Lapoux et al., Phys. Lett. B 658, 198 (2008)

[21] M.D. Cortina-Gil et al., Phys. Lett. B 401, 9 (1997)

[22] A.A. Korsheninnikov et al., Phys. Lett. B 343, 53 (1995)

[23] R.F. Carlson, At. Data Nucl. Data Tables 63, 93 (1996)

[24] A. de Vismes et al., Nucl. Phys. A 706, 295 (2002)

[25] P.D. Kunz and E. Rost, Computational Nuclear Physics, 2, eds. K. Langanke et al. (Springer-Verlag, New York, 1993), p. 88.

[26] C.A. Bertulani, C.M. Campbell, and T. Glasmacher, Comput. Phys. Commun. 152, 317 (2003)

[27] R.J. Glauber, Lectures in Theoretical Physics, 1, eds. W. E. Brittin and G. L. Dunham (Interscience, New York, 1959), p. 315.

[28] P. Mohr, Phys. Rev. C 61, 045802 (2000) 